\title{
Editorial
}

\section{p53: more research and more questions}

\author{
AW Braithwaite ${ }^{*, 1}$ and CL Prives ${ }^{2}$ \\ ${ }^{1}$ Department of Pathology, Dunedin School of Medicine, University of Otago, \\ Great King Street, Box 913, Dunedin, Otago 9001, New Zealand and \\ 2 Department of Biological Sciences, Columbia University, New York, NY, USA \\ * Corresponding author: AW Braithwaite, Department of Pathology, Dunedin \\ School of Medicine, University of Otago, Great King Street, Box 913, Dunedin, \\ Otago 9001, New Zealand. Tel: +64 3479 7165; Fax: + 643479 7136; \\ E-mail: antony.braithwaite@stonebow.otago.ac.nz
}

Cell Death and Differentiation (2006) 13, 877-880.

doi:10.1038/sj.cdd. 4401938

\section{Preamble}

This special review issue of Cell Death and Differentiation is about the tumor suppressor p53. It was prompted by the most recent International p53 Workshop, held in Dunedin, New Zealand, in November 2004. However, it is not exclusively focused on the work presented at that meeting, but rather represents a set of reviews on topics of current interest in the p53 field using the Dunedin meeting as a springboard. To this end, the issue begins with an editorial outlining a brief history and an overview of the p53 field to place the reviews and commentaries that follow in context.

\section{Historical Perspective}

p53 was discovered independently by David Lane ${ }^{1}$ and Arnold Levine $^{2}$ in 1979 as a cellular protein in complex with the large $\mathrm{T}$ antigen (LT) of SV40. Although it took a few years, p53 cDNAs were isolated and cloned ${ }^{3,4}$ and, consistent with its association with LT, p53 was found to cooperate with other oncogene products in in vitro transformation assays. ${ }^{5-8}$ This, combined with several observations that p53 was overexpressed in human cancer cell lines (e.g., Crawford et $a l^{9}{ }^{9}$ ), suggested that p53 was a bona fide oncogene. In addition, there were many in vitro studies that were consistent with this interpretation (e.g., Mercer et al. ${ }^{10}$ ). However, there were also observations that were not consistent, and when Vogelstein and colleagues reported in 1989 that there was frequent 'loss-of-heterozygosity' at the p53 locus (TP53) in a series of human colorectal cancers, ${ }^{11}$ it seemed that maybe p53 was not an oncogene but, in fact, its antithesis, a tumor suppressor gene (TSG) (reviewed by Benchimol and Lane ${ }^{12}$ ). This conclusion was soon confirmed by a number of in vitro studies ${ }^{13}$ and the analysis of many tumors of many different types demonstrated that TP53 was very frequently mutated. Indeed, we now know that mutations in the TP53 gene in human cancers are more common than for any other gene (www.iarc.fr/p53). These data, combined with the observations that mice genetically deficient in TRP53 (the mouse p53 gene) are extremely tumor prone ${ }^{14}$ and that the inherited cancer predisposing syndrome in humans, Li-Fraumeni
(LFS), is due to germline mutations in $T P 53,{ }^{15,16}$ show beyond reasonable doubt that TP53 is a TSG.

Since this paradigm shift of the late 1980s and early 1990s, research on $\mathrm{p} 53$ has been characterized by intense investigations into the p53 signaling pathways, its mechanism(s) of action and how p53 functions as a tumor suppressor. However, despite the wealth of information that has accumulated in this time ( $>36000$ papers worth!), a complete understanding of how p53 functions still remains elusive. Nonetheless, some general patterns have emerged over the years.

\section{The p53 Pathway}

In broad terms, a wide variety of cellular stress signaling pathways engage the p53 network, resulting in marked accumulation and activation of the p53 protein. The most well studied of these to date are the ones due to DNA damage (see review by Lavin and Gueven, this issue) and to aberrant proliferative signaling, although others such as hypoxia are probably of equal importance. Once activated, p53 can function as a sequence-specific transcription factor that can act directly and indirectly to turn on or off various genes that affect cell growth and survival. Additionally, p53 can directly interact with several proteins that influence growth and survival. Specifically, p53 can modulate cell cycle progression, senescence and apoptosis as well as probably playing a direct role in DNA repair and recombination (see Figure 1 for an outline of the p53 pathway). By manipulating these processes, $\mathrm{p} 53$ can prevent tumor formation by reducing the likelihood with which genetic lesions accumulate: lesions that may contribute to tumorigenesis. If normal p53 functions are compromised, cells are frequently predisposed to tumor formation. Reviews in this issue of Cell Death and Differentiation deal with all these aspects of p53 biology.

The process of $p 53$ regulation may be divided broadly into three phases: the activation phase, the effector phase and the outcome phase, although Levine et al. (this issue) subdivide this further. These three phases are discussed below in relation to the reviews.

\section{The Activation Phase}

In order for p53 to induce a measurable biological response after stress signaling, its levels need to reach a certain threshold and its specific activity may also be increased. It is likely that the main target of most signaling pathways is to counteract the ability of $\mathrm{Mdm} 2$, the predominant negative regulator of p53, to hold p53 in check (see review by Marine et al., this issue). This seems often to occur through some kind of direct post-translational modification to the p53 protein, which generally results in p53 protein stabilization, but may also confer some special properties on p53. However, in some cases p53 is stabilized and active even without obvious 


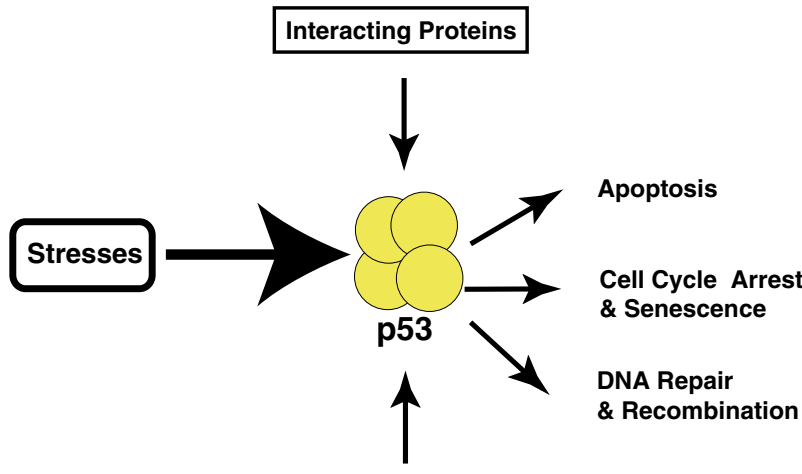

Post-translational modifications

Figure 1 A simplified cartoon of p53 activation and response

post-translational modification. Further, the nature of the modifications depends to some extent on the upstream signals. Some residues appear to be ubiquitously modified in response to different stresses, such as Serine 15 in human p53, ${ }^{17,18}$ whereas others are more restricted, such as Serine 392, which, for example, is not modified in response to doublestrand DNA-damaging agents (Lu et al., ${ }^{19}$ reviewed by Prives and $\mathrm{Hall}^{20}$ ). How important these modifications are for p53 activity is still not clear as there are examples where overexpression of $\mathrm{p} 53$ can circumvent the need for additional modifications and, in some but not all cases, mice with 'modifiable' amino-acid residues mutated were shown to behave similarly to wild-type mice. ${ }^{21,22}$ Most attention has been focused on phosphorylation, particularly of the $\mathrm{N}$ terminus of $\mathrm{p} 53$, although isomerization of several $\mathrm{N}$-terminal amino-acid residues has also been shown to occur. Numerous and varied modifications of residues of the $\mathrm{C}$-terminus have also been documented, including phosphorylation, acetylation and methylation. C-terminal lysines can also be ubiquitylated, sumoylated and neddylated, and each of the above modifications can in some assays affect p53 levels or activity (see e.g., the review by Anderson and Appela ${ }^{23}$ ). Although no single review in this issue specifically focuses on the importance of post-translational modifications in p53 activation, it is nonetheless addressed in reasonable detail by Lavin and Gueven, by Wahl and, to a lesser extent, by other contributors throughout the issue.

\section{The Effector Phase}

Once p53 has been activated, it can then begin eliciting a biological response. Structural data support multiple other lines of evidence that p53 is a sequence-specific transcription factor. A detailed structure-function analysis of p53 and its family members $p 63$ and $p 73$, with respect to $p 53$ functioning as a transcription factor, is provided by Harms and Chen in this issue, as well as new forms of p53 by Murray-Zmijewski et al. (see below). However, there are also data showing that p53 has transcription-independent activities (reviewed by Moll et al. ${ }^{24}$ and Chipuk and Green ${ }^{25}$ ).
With respect to transcriptional regulation, p53 has to 'decide' which genes it is going to activate (or repress), scan $3 \times 10^{9}$ base pairs in the midst of complex chromatin for its binding site(s) and then interact with the core transcriptional machinery to initiate transcription. This is a tall order indeed! The issues involved in this scanning and selection process, and how p53 actually transactivates its target genes, are discussed in three papers in this issue - Laptenko and Prives on transcription, Liu and Kulesz-Martin on scanning, and Kim and Deppert on interactions with DNA. As p53 is so prominent in current cell and cancer biology, it is not surprising that it has been used as a model transactivator by a number of investigators in the transcription field and a number of interesting findings have been made about its interactions with the transcriptional machinery of cells. Still, there are important questions that remain essentially unanswered such as how it selects different promoters in different circumstances, whether it coregulates genes with its family members, $p 63$ and p73, and what is the mechanism by which it represses some genes.

However, while understanding of p53's transcription mechanisms is improving, how p53 makes decisions to do one thing or another, or turn on one gene or another, is far from clear. Post-translational modifications are likely to play roles and such modifications and their impact on $\mathrm{p} 53$ have achieved prominence in the literature. Another way that p53 decides is by virtue of its interactions with other cellular proteins, although the two mechanisms are not mutually exclusive. The list of p53-interacting proteins is large ( $>80$ and growing, see Table 1 in review by Braithwaite et al., in this issue) and so it is difficult to rationalize how $p 53$ can respond and interact with all of them. Probably, these interactions are transient and depend very much on the nature of the upstream signals and on the cell type. Some interacting proteins disable p53 (at least as a transcription factor), but others appear to dictate what p53 can do - that is, they act as cofactors. Some interesting cofactors that may have considerable impact on p53 function are discussed in the review by Braithwaite et al., in this issue, and also by Chipuk and Green.

\section{The Outcomes Phase}

Much attention has been directed towards the two most wellstudied biological outcomes of p53 - induction of cell cycle arrest and apoptosis. Nevertheless, it must also be emphasized that p53 can induce a cellular senescence-like phenotype and may activate DNA repair processes and repress recombination. These outcomes could all potentially contribute to the tumor suppressor activity of p53, although the bulk of evidence suggests that apoptosis is the most important (see review by Johnson and Attardi in particular). However, this may well depend on the nature of the tumor inducer and the tissue type.

The induction of cell cycle arrest predominantly occurs as a result of p53 transactivating the gene CDKN1A that encodes the cyclin-dependent kinase (CDK) inhibitor p21 WAF $1 / \mathrm{CIP} 1$ (reviewed by Prives and Hall ${ }^{20}$ ). This blocks the activity of $C D K s$ in complex with cyclins $D$ and $E$ needed for $G_{1}$ cell cycle progression, and results in $\mathrm{G}_{1}$ arrest. Cell cycle arrest can 
also occur by transactivation of the GADD45 and 14-3-3 $\sigma$ genes. In this issue of Cell Death and Differentiation, there is no one review that focuses on the process of cell cycle arrest by $\mathrm{p53}$. This is in part because it is mentioned frequently throughout the issue, but also because the mechanism of cell cycle arrest is reasonably well understood. Indeed, in the review by Prives and Kulesz-Martin, on how p53 functions as a transcription factor, the $C D K N 1 A$ promoter is frequently used as a model target gene. However, the review by Opferman and Zambetti offers a new perspective on cell cycle checkpoint control.

How p53 facilitates the process of apoptosis is much less clear than for cell cycle arrest. Considerable evidence suggests that again p53 functions as a transcription factor to effect this process; however, identification of the 'key' target gene or genes has been slow coming, partly because there are many putative candidates. Studies discussed here by Chipuk and Green strongly suggest that the members of the $B C L-2$ family are important, particularly the BH3-only members, NOXA and PUMA. Apart from induction of apoptosis by transactivation of specific target genes, there is a growing body of literature suggesting that p53 can directly interact with BH3-only proteins in the cytoplasm as well as in the mitochondrial membrane to facilitate cytochrome $c$ release and apoptosis. The different modes of apoptosis induction are discussed in depth by Chipuk and Green. The mechanism of p53-dependent apoptosis is also discussed in the review on human p53 variants by Murphy.

Another potentially important mode of action for p53 is in the regulation of DNA recombination and genetic instability, which are important for tumorigenesis, and of DNA repair. Wiesmueller, in an extensive review, discusses in depth the evidence that p53 plays a direct role in DNA repair processes and in recombination. With respect to the latter, experiments suggest that p53 can either inhibit or stimulate different kinds of DNA recombination events and that this appears to be independent of its transactivation ability.

\section{Models of p53 Function}

While a vast amount of information has been generated using cell culture and in vitro studies, as well as from an analysis of human tumors (see Royds and lacopetta, this issue), tests of critical hypotheses concerning p53 function can only be carried out in animals. The use of transgenic, knockout and, more recently, knock-in mouse mutants of Trp53 has become a powerful genetic tool to uncover the molecular basis of p53 function and to study its contribution to tumorigenesis and other biological processes. This point is well illustrated in recent studies in which the construction of mice with the equivalent of some of the common human p53 tumor mutants has generated a tumor spectrum phenotype similar to that of LFS patients, whereas the knockout mouse, while still demonstrating that p53 is a tumor suppressor, generated a tumor spectrum very different from that in the human context. At least two reviews in this issue discuss mouse models of p53 function and their contribution to tumorigenesis. In particular, Johnson and Attardi discuss how the use of mutant mice helps us understand the key components of signaling to p53 and what biochemical properties of p53 are required for tumor suppressor activity, as does Wahl. Moreover, these authors highlight possible roles for p53 in regulating mouse development, something that only can be determined using animal models.

In addition, Haupt et al. along with Lu and Abrams outline the contributions that non-mammalian organisms have lent to our understanding of the p53 network, particularly in apoptosis and the impact of p53 on lifespan. The latter point being of special interest as normal TP53 variants (polymorphisms) may play an important role in longevity (see review by Murphy).

\section{p53 - Predisposition, Disease and Therapy}

How p53 prevents tumor formation and whether mutant forms of p53 can contribute to disease progression is important to understand, particularly with regards to developing new treatment regimens for cancer. However, an area that until recently has been largely ignored concerns the contribution of natural TP53 polymorphisms and the p53 pathway to tumor susceptibility. Some polymorphisms are quite frequent while others are infrequent. The review by Murphy, a pioneer in this field, discusses the importance of two polymorphisms, Pro72Arg in human p53 and an HDM2 promoter variant, to tumor predisposition. She also speculates on why some allelic variants might confer a selective advantage.

Following this, Royds and lacopetta discuss a wide variety of literature concerning TP53 status (i.e., whether TP53 is wild type or mutant and, if so, what are the mutations) in common human malignancies, particularly colorectal cancers and glioblastomas, but they also discuss LFS in some depth. Considerable attention is paid to the clinical impact of TP53 mutation detection. For example, they review evidence investigating whether TP53 mutation detection has prognostic value and whether or not it is useful in predicting patient response to therapy. In the final part of this review, the authors discuss the contribution of dysregulation of the $\mathrm{p} 53$ pathway to non-neoplastic diseases such as atherosclerosis and neurological syndromes. This is also discussed by Levine et al., who also provide compelling evidence that the status of the HDM2 gene, notably of its newly discovered polymorphism, can have a major impact on cancer occurrence and outcome.

As is clear from the extensive analysis of tumors reviewed by Royds and lacopetta, the p53 pathway is probably impaired in all human malignancies. As such, $\mathrm{p} 53$ and the $\mathrm{p} 53$ pathway would appear to be excellent targets for the development of novel therapeutics. Wiman discusses the targeting of the p53 pathway for therapeutic intervention in the treatment of cancers. There appear to be numerous strategies: 'activation' of p53 in tumors containing a genetically wild-type TP53 gene, but an apparently inert protein (or replacement of p53 function with an exogenous gene); the 'reactivation' of mutant p53 protein by presumably inducing changes in conformation; and the construction of agents that target a defective $\mathrm{p} 53$ pathway. All approaches are discussed in this review. 


\section{Novel p53 Isoforms}

And just to make things more complicated than they already were, Murray-Zmijewski et al. (in this issue) discuss their discovery that p53 is not alone. ${ }^{26}$ Until the late 1990 s, p53 was the sole member of its own family. Then, p63 and p73 were discovered, as were isoforms of both proteins (reviewed by Melino et $a .^{27}$ ). On the basis of an analogy with these other family members, Bourdon and co-workers searched for and found a number of isoforms of p53 itself. Together with another isoform recently reported from the Deppert laboratory, ${ }^{28}$ these new p53 family members are set to have a major impact in the p53 field.

\section{p53 - The Future}

Finally, this special issue of Cell Death and Differentiation is completed with an insightful commentary by Levine et al. In this review, the authors look beyond the role of p53 just as a tumor suppressor to consider the very real possibility that p53 is a 'nodal' stress point and is therefore important in preventing cellular dysfunction in response to stresses that do not lead to genetic lesions, and are therefore not obviously linked to tumorigenesis. Recent data suggesting p53's involvement in atherosclerosis and possibly in neurological disorders provide support for this new paradigm of p53 function.

While a decade ago it was possible to write a comprehensive single review on $\mathrm{p} 53$, the multitude and complexity of findings since then in this area have made this virtually impossible. Therefore, it is hoped that the set of review articles in this issue of Cell Death and Differentiation will provide in aggregate a timely and useful summation of the key findings on p53 to date as well as provide directions for new research in this still exciting area.

1. Lane DP and Crawford LV (1979) Nature 278: 261-263.

2. Linzer DI and Levine AJ (1979) Cell 17: 43-52.

3. Oren M and Levine AJ (1983) Proc. Natl. Acad. Sci. USA 80: 56-59.

4. Matlashewski G et al. (1984) EMBO J. 3: 3257-3262

5. Jenkins JR, Rudge K and Currie GA (1984) Nature 312: 651-654.

6. Eliyahu D et al. (1984) Nature 312: 646-649.

7. Parada LF et al. (1984) Nature 312: 649-651.

8. Wolf D, Harris N and Rotter V (1984) Cell 38: 119-126.

9. Crawford LV et al. (1981) Proc. Natl. Acad. Sci. USA 78: 41-45.

10. Mercer WE et al. (1982) Proc. Natl. Acad. Sci. USA 79: 6309-6312.

11. Vogelstein B et al. (1989) Science 244: 207-211.

12. Lane DP and Benchimol S (1990) Genes Dev. 4: 1-8.

13. Finlay CA, Hinds PW and Levine AJ (1989) Cell 57: 1083-1093.

14. Donehower L et al. (1992) Nature 356: 215-221.

15. Malkin D et al. (1990) Science 250: 1233-1238.

16. Srivastava $S$ et al. (1990) Nature 348: 747-749.

17. Canman CE et al. (1998) Science 281: 1677-1679.

18. Banin S et al. (1998) Science 281: 1674-1677.

19. Lu H et al. (1998) Proc. Natl. Acad. Sci. USA 95: 6399-6402.

20. Prives $C$ and Hall PA (1999) J. Pathol. 187: 112-126.

21. Krummel KA et al. (2005) Proc. Natl. Acad. Sci. USA 102: 10188-10193.

22. Thompson T et al. (2004) J. Biol. Chem. 279: 53015-53022.

23. Appela E and Anderson CW (2001) Eur. J. Biochem. 268: 2764-2772.

24. Moll UM et al. (2005) Curr. Opin. Cell Biol. 17: 631-636.

25. Chipuk JE and Green DR (2003) J. Clin. Immunol. 23: 355-361.

26. Bourdon JC et al. (2005) Genes Dev. 19: 2122-2137.

27. Melino G et al. (2003) Trends Biochem. Sci. 28: 663-670.

28. Rohaly G et al. (2005) Cell 122: 21-32. 